\title{
Estimating collectable solar energy by partially shaded collectors using custom-designed charts and tables: demonstration for typical Zimbabwe locations
}

\author{
T Hove \\ Department of Mechanical Engineering, University of Zimbabwe \\ D Chipfunhu \\ Department of Metallurgical Engineering, University of Zimbabwe
}

\begin{abstract}
In this paper, an approach for the estimation of insolation available to a solar energy collector that may be shaded temporally by external horizon obstructions is described. This approach uses, in place of complicated mathematical expressions or sophisticated specialized computer software, a set of custom-designed charts and tables, and a simple equation, to evaluate the temporal radiation income to the solar collector. The solar position diagram or sun-chart is first used to determine shading status, and then a package of charts and tables is used to evaluate a suitable tilted-plane sky model for collectable insolation. The approach is convenient for use by the average solar energy designer who might not have background training in the intricacies of solar geometry and meteorology. Although in the present case, the charts and tables have been designed to be applicable in Zimbabwe and for the standard flat plate configuration - equator facing and tilt equal to latitude, they can also be produced for any other location and collector configuration. A major approximation in the procedure was to neglect the effects of shading on diffuse radiation availability. The resultant error was analysed and was concluded negligible for reasonably likely cases involving low-tilt collectors.
\end{abstract}

Keywords: solar geometry, charts collector, shading analysis

\section{Introduction}

Estimation of collectable insolation is a necessary first step in the prediction of energy delivery potential of solar energy systems. Installation sites for solar energy collectors are almost invariably shaded from the sun's beam by external objects such as the horizon line and other obstructions such as trees and buildings, at certain times of the day, during certain months of the year, resulting in reduced radiation capture by the collectors. Besides the challenging task of resolving daily global radiation temporally and resolving it into its beam and diffuse components, calculation of collectable insolation requires prediction of the temporal shading status of the collector site. The calculations involved in such an exercise are tedious and require a considerable amount of specialised expertise. Analytical models, e.g. (Dennis 2002), can be developed, for analysing insolation on shaded collectors, but their use requires special background training in solar geometry and meteorology. Special solar energy computer software packages such as TRNSYS (Klein 2002) are very costly and ordinarily unaffordable by solar energy designers, particularly in developing countries. The use of custom-designed tools such as charts and tables may circumvent these problems, and can allow both professional and amateur solar designers to easily evaluate the collectable solar radiation by solar electrical and thermal modules, passive solar homes, greenhouses, and other solar devices.

At a given location (latitude), the shading status of a collector site depends on the angular position of the sun relative to the site, in comparison with the angular positions of the different obstructions, including the horizon line, in the proximity of the site. The position of the sun relative to an observer on earth is defined by two angles; the solar azimuth and altitude angles (Rabl 1985). The solar azimuth angle, $\alpha_{s}$, is the angular displacement from north (for Southern hemisphere locations) of the projection of the sun's beam on the horizontal plane. It measures the sun's location in degrees east or west of due north, with displacements east of north defined as positive. The solar altitude angle, $\alpha_{\mathrm{s}}$, is the angle between the horizontal and the line join- 
ing the sun and the observer. A plot of $\alpha_{s}$ against $\gamma_{s}$ is called a sun chart (Crawford 1995), or a solar position diagram (Duffie \& Beckman 1991). It can be used as a convenient tool to analyse shading of a chosen collector site by nearby high-rise objects or the horizon line. However for complete evaluation of collectable insolation by a collector at the site, the sun chart needs to be complemented by other information, for instance, the relationship between solar and civil time, the hourly distribution of daily solar radiation, the diffuse fraction of global radiation, and other factors.

This paper demonstrates the use of customdesigned or 'home-made' charts and tables to estimate the amount of insolation collectable by a solar collector, at a site that is partly shaded by external obstructions. The charts and tables are intended for use by technicians or engineers without background training in solar geometry and energy meteorology. Although the approach can be applied for any location, and for any collector configuration, the charts and tables in this paper are custom-designed for Zimbabwe locations, and for the common flat plate collector configuration - equator facing with tilt angle equal to latitude.

\section{The sun chart}

Figure 1 shows a sun chart or solar position diagram for Harare, latitude $17.8^{\circ} \mathrm{S}$. The chart is a plot of the sun's path across the sky during the year. It shows the position of the sun at any time on any day of the year through a plot of the solar altitude angle against the solar azimuth angle. The sun chart shown in Figure 1 was deliberately made to map out the path of the sun on mean radiation days of each month of the year as recommended by Klein (1977). The mean day of the month is the day with extraterrestrial (outside atmosphere) solar radiation closest to the monthly mean. Also on the sun chart, iso-time curves connect the solar path curves to provide time-of-day information. These curves are drawn an hour apart, with solar noon being along the $y$-axis of the sun chart.

Some simple but important observations can be deduced from the sun chart. These include: the position of the sun at any time of the day and any day (or month) of the year; the sunrise/sunset times and directions for each month (in fact for each day); the angle of incidence of beam radiation on a horizontal plane (the complement of the altitude angle); and other deductions.

The times given on the sun chart are in terms of solar time instead of clock or civil time. The conversion between solar time and clock time can be done using mathematical functions of longitude and day of the year. It also depends on whether or not daytime saving is practiced. For locations in Zimbabwe (longitude 25 to $33^{\circ}$ East, Pretoria standard time), and where daytime saving is not practiced, the necessary range of corrections have been calculated and tabulated in Table 1 . To get solar time (which is necessary in using Figure 1) for a given clock time, the correction given in Table 1 should be added to clock time.

For example, to determine the angular position of the sun at Harare (longitude $32.7^{\circ}$ East) at 10:34 am, clock time, on 15 October, Table 1 is used to convert clock time to solar time. After interpolation, the correction can be seen to be approximately 26 minutes hence the corresponding solar time is 11:00 am. Figure 1 is then used for 11:00 am on October 15 , from which we read that the sun is $63^{\circ}$ east of north (azimuth angle $63^{\circ}$ ), and is at an altitude angle of $73^{\circ}$. Having located the position of

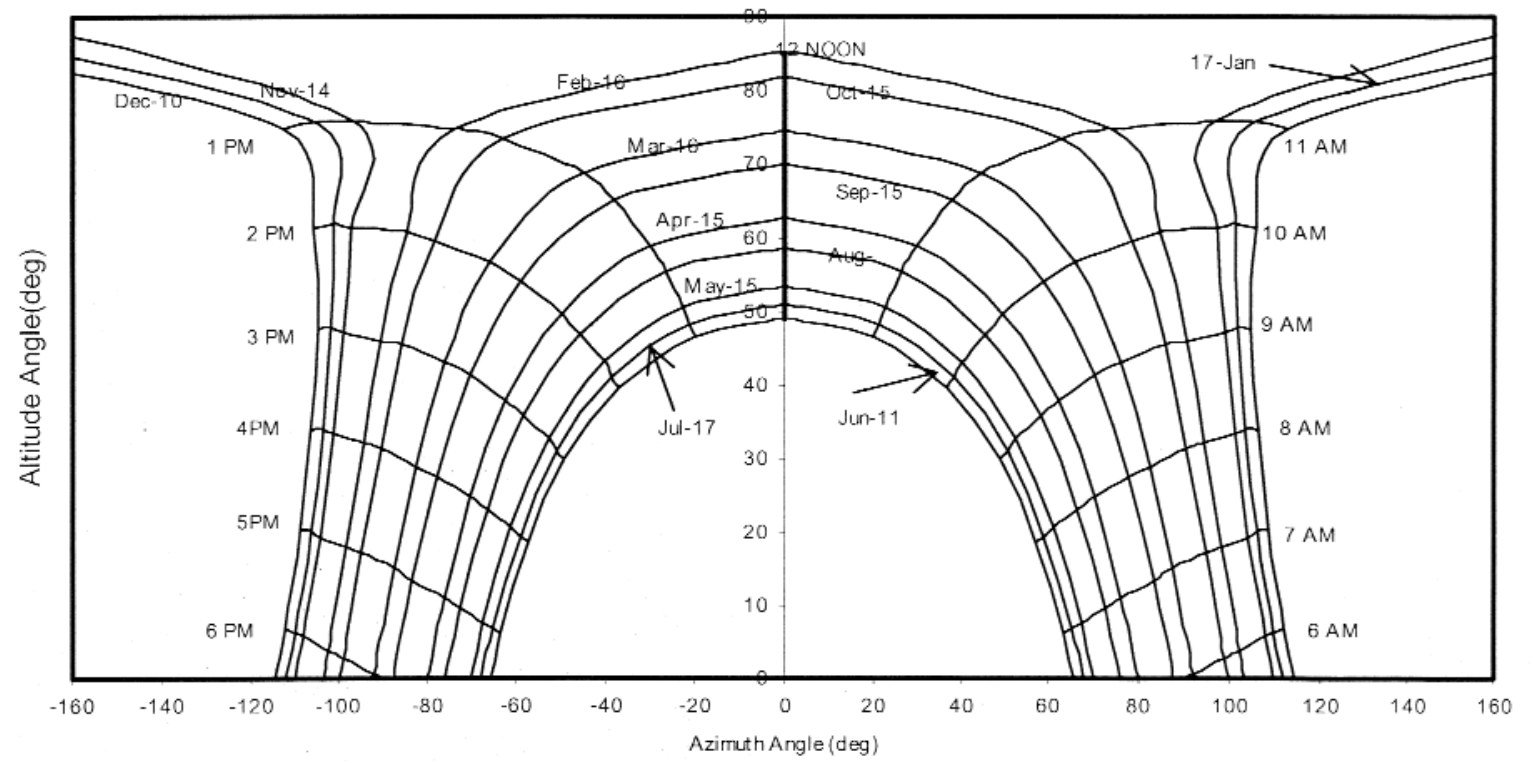

Figure 1: Sun chart for Harare, latitude $17.8^{\circ} \mathrm{S}$ 
Table 1: Correction from standard time to solar time for Zimbabwean locations

\begin{tabular}{lcccccccccccc}
\hline $\begin{array}{l}\text { Longitude } \\
\text { (deg. east) }\end{array}$ & \multicolumn{10}{c}{$\begin{array}{c}\text { Correction (minutes) } \\
\text { (Solar time = clock time + correction) }\end{array}$} \\
\hline & Jan 17 & Feb 16 & Mar 16 & Apr 15 & May 15 & Jun 11 & Jul 17 & Aug 16 & Sep 15 & Oct 15 & Nov 14 Dec 17 \\
\hline 25 & -30 & -35 & -29 & -20 & -16 & -20 & -26 & -24 & -14 & -5 & -5 & -14 \\
\hline 26 & -26 & -31 & -25 & -16 & -12 & -16 & -22 & -20 & -10 & -1 & -1 & -10 \\
\hline 27 & -22 & -27 & -21 & -12 & -8 & -12 & -18 & -16 & -6 & 3 & 3 & -6 \\
\hline 28 & -18 & -23 & -17 & -8 & -4 & -8 & -14 & -12 & -2 & 7 & 7 & -2 \\
\hline 29 & -14 & -19 & -13 & -4 & 0 & -4 & -10 & -8 & 2 & 11 & 11 & 2 \\
\hline 30 & -10 & -15 & -9 & 0 & 4 & 0 & -6 & -4 & 6 & 15 & 15 & 6 \\
\hline 31 & -6 & -11 & -5 & 4 & 8 & 4 & -2 & 0 & 10 & 19 & 19 & 10 \\
\hline 32 & -2 & -7 & -1 & 8 & 12 & 8 & 2 & 4 & 14 & 23 & 23 & 14 \\
\hline 33 & 2 & -3 & 3 & 12 & 16 & 12 & 6 & 8 & 18 & 27 & 27 & 18 \\
\hline
\end{tabular}

the sun relative to an observer (solar collector) at Harare on the chosen day and time, the angle of incidence of the solar beam on an unobstructed solar collector can be calculated if its orientation (tilt and azimuth) is known - an important step in determining the collectable solar energy.

Constructing the sun-chart from first principles of course requires considerable expertise in solar geometry, but special computer software, e.g. SUNCHART (Crawford 1995), may be used. Also, as was done in the present case, the altitude and azimuth angles for different times and days can be calculated using standard formulae, e.g. (Rabl 1985), and plotted in a spreadsheet package, for example Excel or similar.

\section{Using the sun-chart to analyse shading of a collector site}

The sun chart can conveniently be used to predict shading of a collector site by nearby high-rise objects or the horizon line, on any given day, at any given time. Measuring or calculating the bearing and altitude angles of all horizon obstructions, and plotting this information on a sun chart, enables one to summarize the shading status of site for the entire year.

As an example, Figure 2 shows a collector site located at X, in the vicinity of a tall flat-roofed, twowinged building, and a relief feature such as a hill. The altitude versus azimuth angle (relative to $\mathrm{X}$ ) of points such as $A, B$, etc, on the building skyline, and
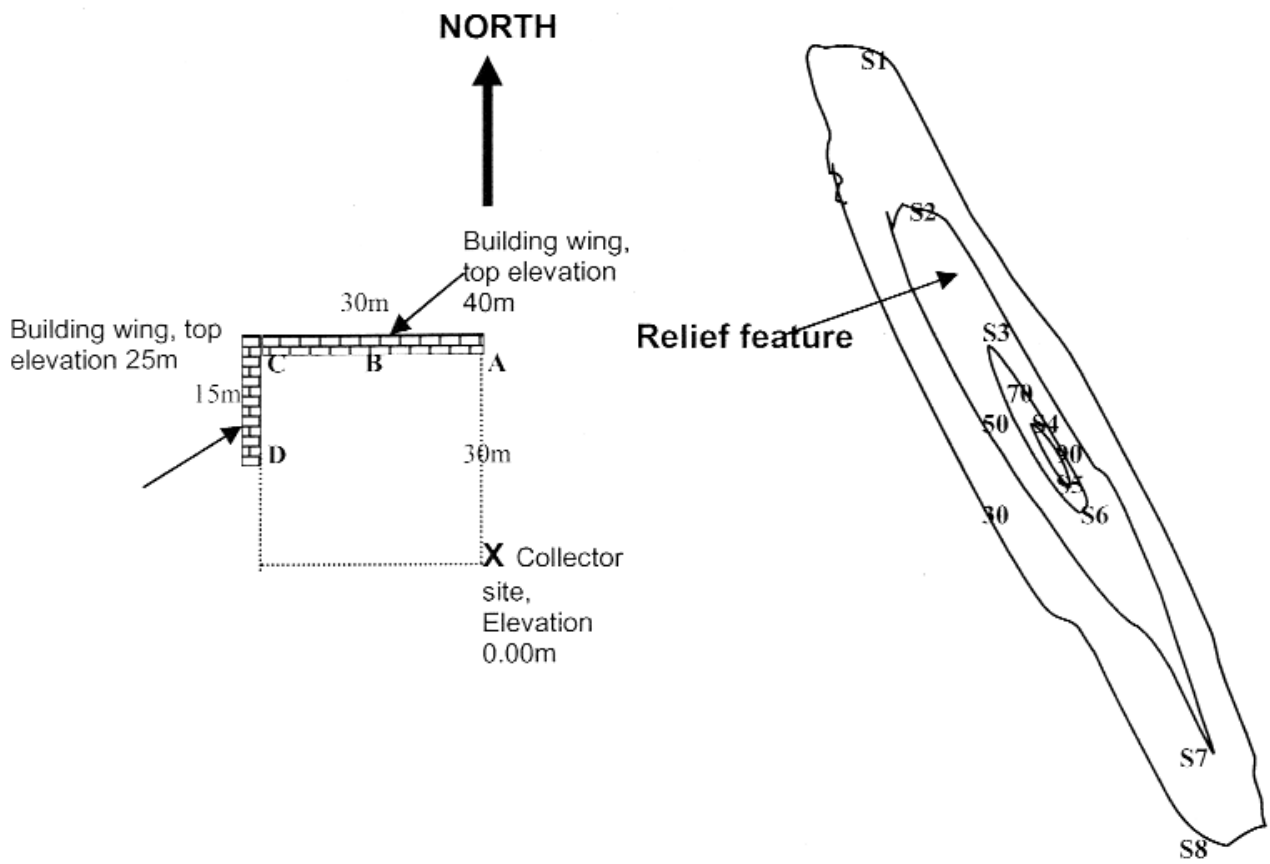

Figure 2: Location plan of a collector site 


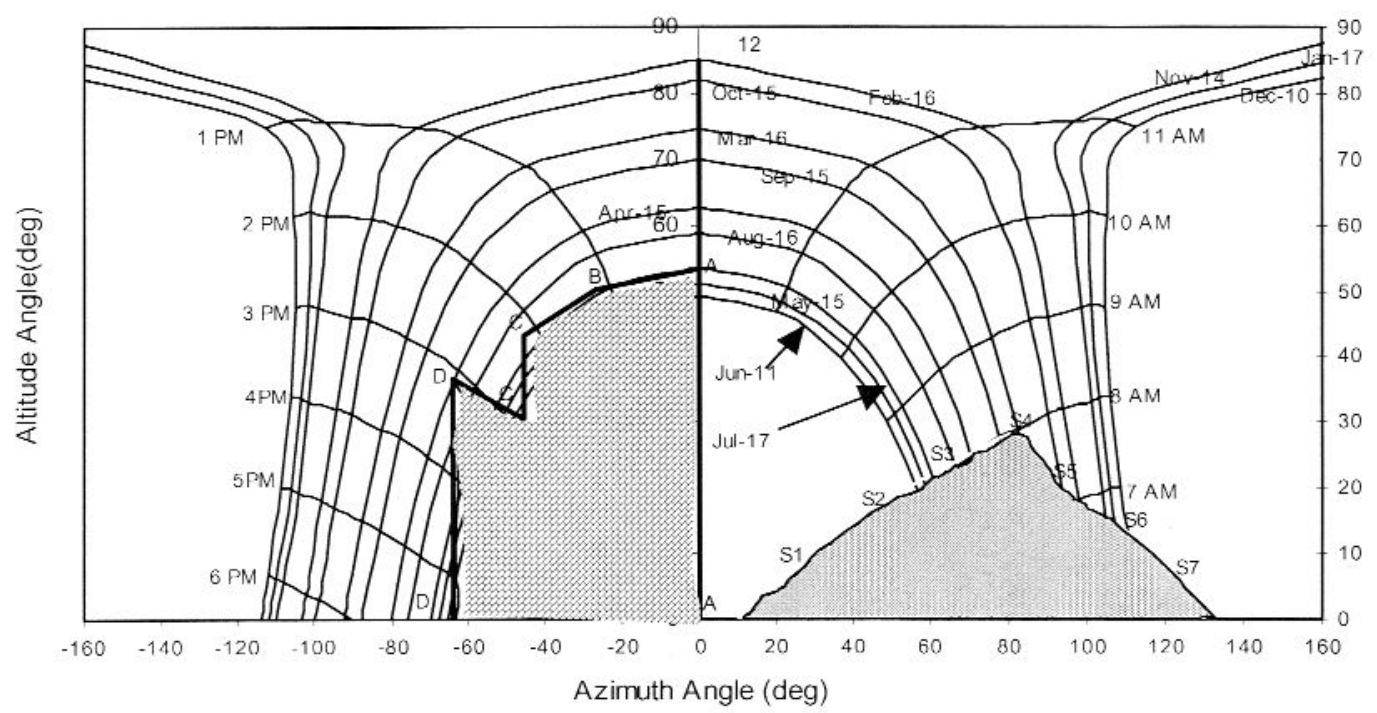

Figure 3: Shading mask superimposed on sun chart

$\mathrm{S} 1, \mathrm{~S} 2$, etc, on the hill skyline, can be plotted on the sun chart as shown in Figure 3. The shaded area shown under the resultant plots is called a shading mask. It is a demarcation(s) on the solar chart for the portions of the sky that are obstructed from sunlight by an object(s) from the observer's eye (collector site), and shows the time / day combinations for this blocking off of sunlight. In this case, it can be read that the hill shades the collector site from sunrise to about $8 \mathrm{am}(7 \mathrm{am})$ in most winter months (in summer months). The building shades the site only during the mid-afternoon hours in winter months.

This information, together with knowledge of the daily solar radiation and its hourly distribution, is of great importance in estimating the available insolation (incident solar radiation) at the collector site, and hence on the collector, as shown in subsequent sections.

\section{Estimating collectable insolation for an un-shaded collector}

For an un-shaded collector, tilted at an angle â to the horizontal, the available hourly insolation, $I_{\text {coll }}$, is given by the tilted-plane sky model of Liu and Jordan (1963):

$$
I_{\text {coll }}=\left(I_{h}-I_{d}\right) R_{b}+F_{d} I_{d}
$$

In equation (1) $I_{h}$ and $I_{d}$ are respectively the global and diffuse hourly irradiation, hence $\left(I_{h}-I_{d}\right)$ is the beam radiation on a horizontal plane. $R_{b}$ is the ratio of beam irradiation on a tilted un-shaded collector to that on a horizontal surface, and $F_{d}$ is the view factor from the collector to the sky- the fraction of the sky dome 'seen' by the collector.

The ratio $R_{b}$ varies with collector orientation (tilt and azimuth), season (day of the year) and time of the day, as well as latitude of the location. Although analytical expressions exist for $R_{b}$ in terms of these variables, following the general approach of this paper, custom-designed charts may be used instead. For instance, for the standard configuration of an equator-facing flat plate collector with tilt angle equal to latitude, monthly average hourly values of $R_{b}$ for latitude $15^{\circ} \mathrm{S}$ and $23^{\circ} \mathrm{S}$ (covering Zimbabwe) have been plotted in Figure 4 . The $R_{b}$ values vary by less than $20 \%$ across Zimbabwe and values for intermediate latitudes may be obtained accurately enough through interpolation. Values of $R_{b}$ are symmetrical about solar noon, so that afternoon values can be easily deduced from the given morning values.

The factor $F_{d}$ for a flat plate collector is equal to $1 / 2[1+\cos \beta]$ and has been plotted on Figure 5 for $\beta$ ranging 0 to $90^{\circ}$.

To complete the evaluation of Equation 1, we need the meteorological inputs, $I_{h}$ and $I_{d}$. The problem with this step arises from the difficulty in obtaining meteorological solar radiation data that is resolved on an hourly basis and also disaggregated into its beam and diffuse components. The most widely available solar radiation data is only the daily total or global irradiation on a horizontal plane, $H_{h}$. For long-term estimates of available insolation, the long-term monthly average of $H_{h}$ is an appropriate start. Starting with only the monthly average global radiation, the monthly average values of $I_{h}$ and $I_{d}$ can be determined in the following way.

First, the monthly average daily diffuse radiation, $H_{d}$, is determined from an empirical correlation of the ratio $H_{d} / H_{h}$ with the so-called monthly average clearness index, $K$. The clearness index is the ratio of daily global radiation on a horizontal plane at the earth's surface, $H_{h}$, to the daily extraterrestrial radiation on a horizontal plane, $H_{o}$, i.e. $K$ $=H_{h} / H_{o}$. Monthly average values of $H_{o}$, computed using the standard formula given in Duffie \& 


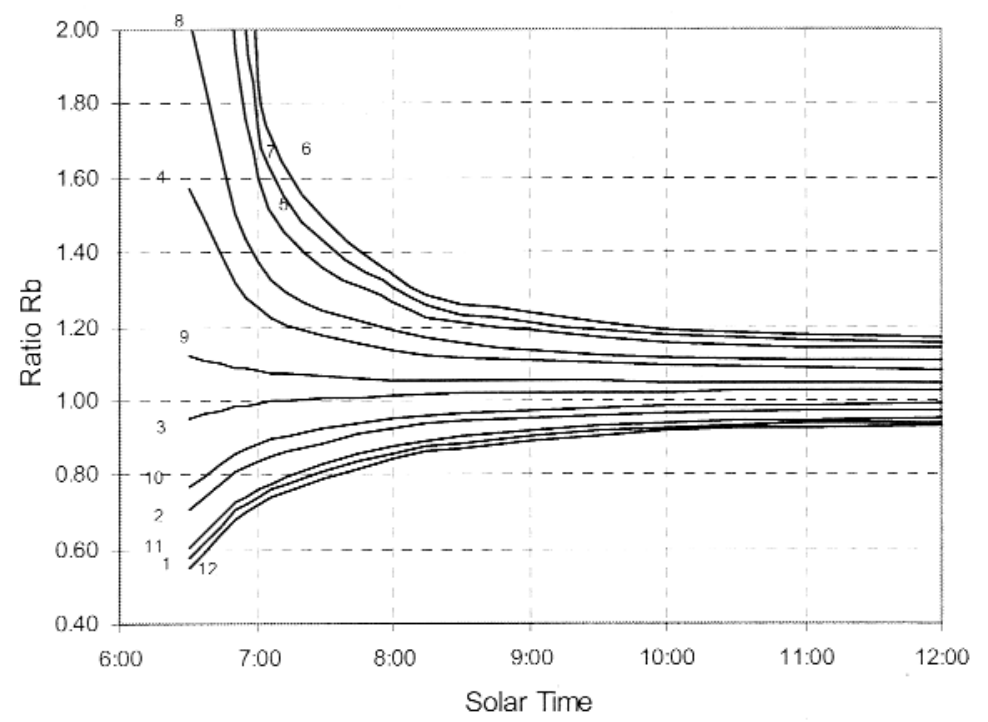

Figure 4a: Ratio $R_{b}$ for latitude $15^{\circ} S$ for a north-facing collector with tilt angle equal to latitude

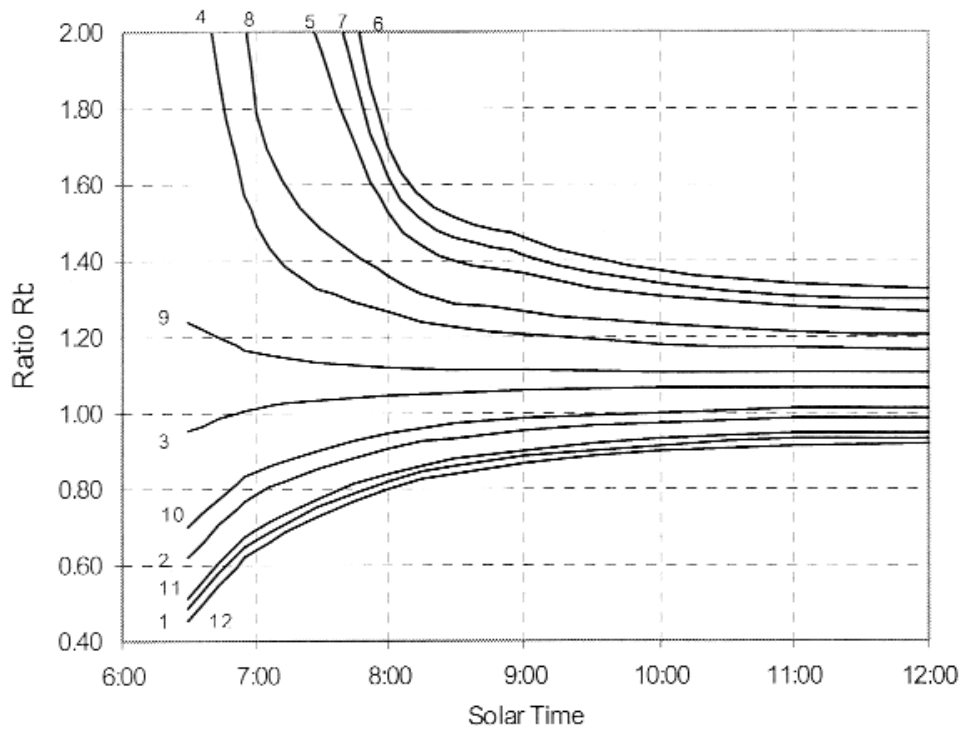

Figure 4b: Ratio $R_{b}$ for latitude $23^{\circ} S$ for a north-facing collector with tilt angle equal to latitude

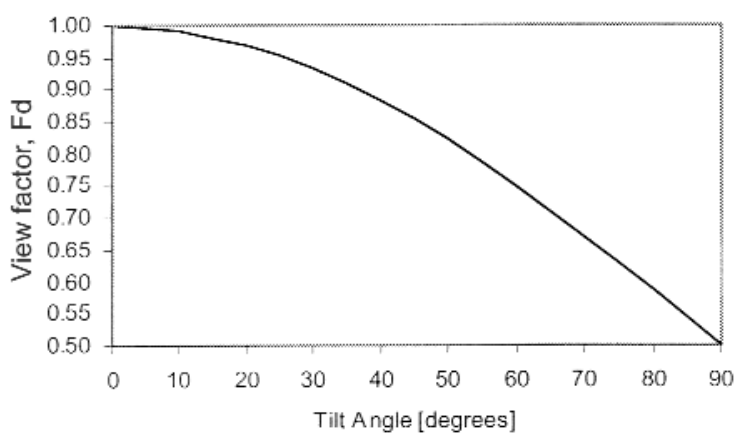

Figure 5: View factor $F_{d}$ versus tilt angle 


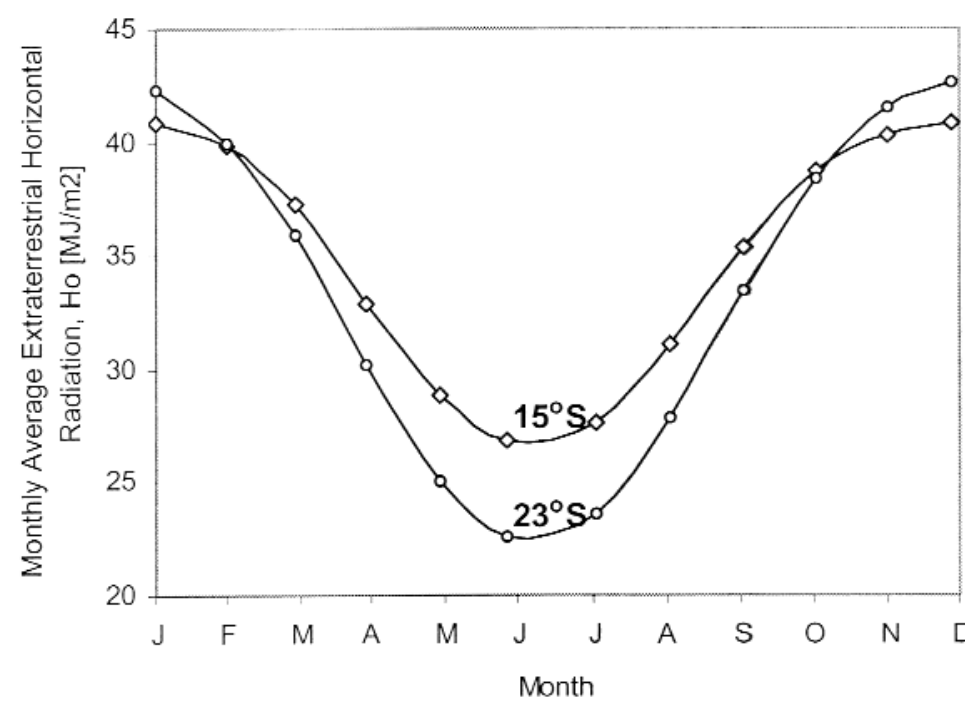

Figure 6: Monthly average daily extraterrestrial irradiation on a horizontal plane for latitudes 15 and $23^{\circ} \mathrm{S}$

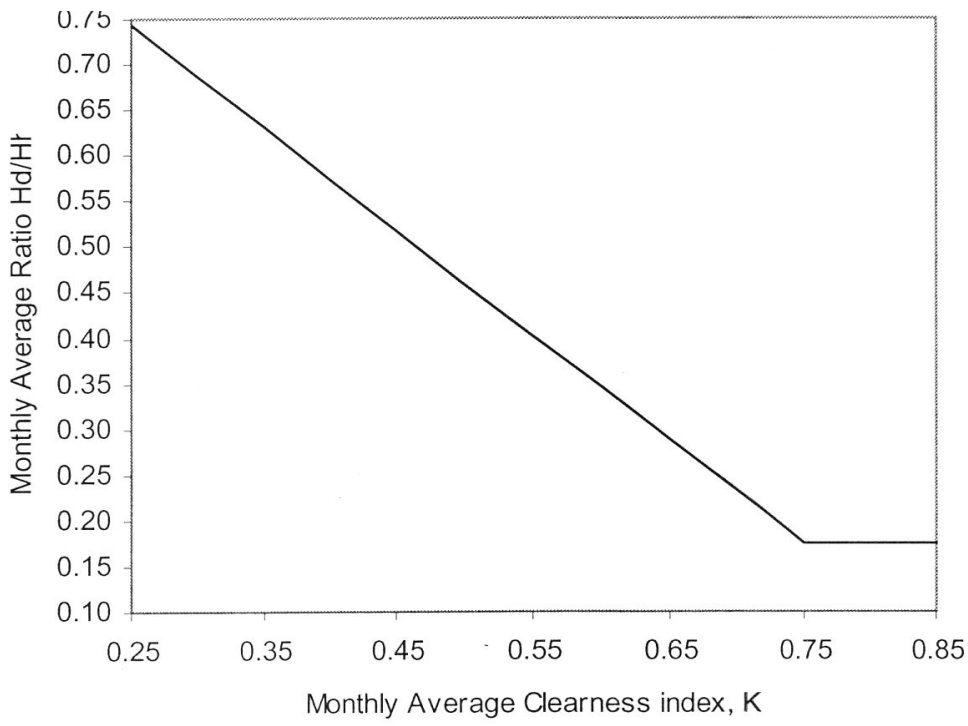

Figure 7: Correlation of the monthly average daily diffuse ratio with monthly average clearness index for Zimbabwe
Beckman (1991), are plotted in Figure 6 for latitudes 15 and $23^{\circ}$ South. Many climate-specific $H_{d} / H_{h}-K$ correlations have been developed by different authors, e.g. Page (1961), Erbs et al (1982), Collares-Pereira \& Rabl (1979), etc. In the case of Zimbabwe, the $H_{d} / H_{h}-K$ correlation developed by Hove and Goettsche (1999), which has been plotted in Figure 7, may be used for all locations in the country.

So, starting only with the meteorologically value of monthly average daily global radiation, $H_{h}$, the monthly average clearness index is calculated as $K$ $=H_{h} / H_{o}$, with $H_{o}$ read from Figure 6 for the month and latitude in question. With this value of $K$ the ratio $H_{d} / H_{h}$ can be read from Figure 7 , and multiplying by $H_{h}$ yields the monthly average daily diffuse irradiation $H_{d}$.

Next, the daily global and diffuse radiation values, $H_{h}$ and $H_{d}$, respectively, have to be resolved into the corresponding hourly values, $I_{h}$ and $I_{d}$ of Equation 1. This is achieved by the use of the conversion factors $r_{h}$ and $r_{d}$ of Collares-Pereira \& Rabl (1979). The factors $r_{h}$ and $r_{d}$ represent the fraction of monthly average daily global and diffuse radiation, respectively, received in a given hour, i.e. $I_{h}=$ $r_{h} H_{h}$ and $I_{d}=r_{d} H_{d}$. They are functions of both day length (therefore day or month of the year) and hour of the day.

Table 2 lists monthly average values of $r_{h}$ and $r_{d}$ for latitude 19S (central Zimbabwe). The values of $r_{h}$ and $r_{d}$ vary by less than $4 \%$ across Zimbabwe, so that using Table 2, for all locations in Zimbabwe, may commit a maximum of only $2 \%$ error. Table 2 , together with Figures 4, 5, 6 and 7 enable the complete evaluation of Equation 1 for Zimbabwe locations. Similar custom-designed charts and tables for calculating collectable insolation may be made for specific use in other regions.

Table 2: Conversion factors, $r_{h}$ and $r_{d}$, of daily to hourly monthly average radiation, at latitude $19^{\circ} S$

\begin{tabular}{l|ccccccc|ccccccc}
\hline$M$ & \multicolumn{5}{|c|}{ Factor $r_{d}$ for solar hour centred at: } & \multicolumn{5}{c}{ Factor $r_{h}$ for solar hour centred at: } \\
\hline & 12 noon & $11 / 13 h r s$ & $10 / 14 h r s$ & $9 / 15 h r s$ & $8 / 16 h r s$ & $7 / 17 h r s$ & $6 / 18 h r s$ & 12 noon & $11 / 13 h r s$ & $10 / 14 h r s$ & $9 / 15 h r s$ & $8 / 16 h r s$ & $7 / 17 h r s$ & $6 / 18 h r s$ \\
\hline Jan & 0.122 & 0.118 & 0.107 & 0.090 & 0.068 & 0.042 & 0.014 & 0.132 & 0.127 & 0.111 & 0.088 & 0.061 & 0.034 & 0.010 \\
\hline Feb & 0.125 & 0.121 & 0.110 & 0.091 & 0.067 & 0.039 & 0.009 & 0.136 & 0.130 & 0.113 & 0.089 & 0.060 & 0.031 & 0.006 \\
\hline Mar & 0.130 & 0.125 & 0.113 & 0.092 & 0.066 & 0.035 & 0.002 & 0.141 & 0.134 & 0.116 & 0.089 & 0.058 & 0.027 & 0.001 \\
\hline Apr & 0.135 & 0.130 & 0.116 & 0.093 & 0.064 & 0.029 & 0.000 & 0.146 & 0.139 & 0.119 & 0.089 & 0.055 & 0.022 & 0.000 \\
\hline May & 0.140 & 0.135 & 0.119 & 0.094 & 0.061 & 0.022 & 0.000 & 0.152 & 0.144 & 0.121 & 0.088 & 0.051 & 0.016 & 0.000 \\
\hline Jun & 0.143 & 0.137 & 0.121 & 0.094 & 0.059 & 0.019 & 0.000 & 0.154 & 0.146 & 0.122 & 0.088 & 0.050 & 0.013 & 0.000 \\
\hline Jul & 0.142 & 0.136 & 0.120 & 0.094 & 0.060 & 0.021 & 0.000 & 0.153 & 0.145 & 0.122 & 0.088 & 0.050 & 0.015 & 0.000 \\
\hline Aug & 0.137 & 0.132 & 0.117 & 0.094 & 0.063 & 0.026 & 0.000 & 0.149 & 0.141 & 0.120 & 0.089 & 0.053 & 0.020 & 0.000 \\
\hline Sep & 0.132 & 0.127 & 0.114 & 0.093 & 0.065 & 0.033 & 0.000 & 0.143 & 0.136 & 0.117 & 0.089 & 0.056 & 0.025 & 0.000 \\
\hline Oct & 0.127 & 0.123 & 0.111 & 0.092 & 0.067 & 0.038 & 0.007 & 0.137 & 0.131 & 0.114 & 0.089 & 0.059 & 0.030 & 0.005 \\
\hline Nov & 0.123 & 0.119 & 0.108 & 0.091 & 0.068 & 0.041 & 0.013 & 0.133 & 0.128 & 0.112 & 0.088 & 0.061 & 0.033 & 0.009 \\
\hline Dec & 0.121 & 0.117 & 0.107 & 0.090 & 0.068 & 0.043 & 0.015 & 0.131 & 0.126 & 0.111 & 0.088 & 0.062 & 0.035 & 0.011 \\
\hline
\end{tabular}




\section{Estimation of collectable insolation for partially shaded collectors}

For the case of a collector that is shaded by external obstructions during a given hour, the beam component of radiation, $I_{h}-I_{d}$ in Equation 1, is eliminated. The fraction of the sky seen by the collector (view factor) is somewhat reduced depending on the aspect of the obstructing object relative to the collector. Although it is possible to calculate the exact effect of external shading on the view factor, for example, by utilizing the crossed-string method of Hottel and Sarofim (1967), it is difficult to generalize this for all perceivable obstruction shapes and aspects. However, since diffuse radiation generally comprises a small fraction of total radiation, neglecting the effect on the view factor will not cause much error in overall insolation estimation, especially so for lowly tilted collectors (almost upward-facing) as should be the case with low latitudes (see Appendix 1). With these considerations, the insolation received during an hour when the collector site is shaded, is given by:

$$
I_{\text {coll,shaded }}=(1-s)\left(I_{h}-I_{d}\right) R_{b}+F_{d} I_{d}
$$

In Equation (2), the variable $s$ represents the fraction of the hour in question when the collector site is shaded as determined from Figure 3 .

By making use of the sun-chart, the hours or part-hours of the day in a given month when the collector site is shaded and can be identified, enabling the calculation of daily collectable insolation from Equation 1 or 2 appropriately. For example, for the collector site in Figure 2 the daily col- lectable insolation by a north-facing collector with tilt equal to latitude, for the month of June, at Harare, can be calculated as follows. Given that the monthly average daily irradiation on a horizontal plane, $H_{h}$, is $16.78 \mathrm{MJ} / \mathrm{m}^{2}, H_{o}$ can be estimated from Figure 6 to be about $25 \mathrm{MJ} / \mathrm{m}^{2}$, and the clearness index calculated to be 0.67 . From Figure 7 , the diffuse ratio, $H_{d} / H_{h}=0.27$ for this value of $K$, hence the monthly average daily diffuse radiation $H_{d}$ is $4.53 \mathrm{MJ} / \mathrm{m}^{2}$. From here, the calculation of collectable isolation proceeds as shown in Table 3, which is essentially self-explanatory. However, it should be noted that near sunrise/sunset, the ratio $R_{b}$ is very large and is not shown in Figure 4. For hours near sunrise the highest $R_{b}$ value given in Figure 4 (2.00 in the present case) may be used without any meaningful error in estimating daily collectable insolation, because only a small fraction of daily radiation is attributable to these hours.

In the present example, the un-shaded collector receives $20.30 \mathrm{MJ} / \mathrm{m}^{2}$ per day, while the occasionally shaded collector receives only $12.04 \mathrm{MJ} / \mathrm{m}^{2}$ during the same period. A significant $40 \%$ reduction in collectable insolation has resulted due to temporally varying shading. This demonstrates the importance of carrying out a shading analysis where temporal external shading of collector sites is suspected.

\section{Conclusion}

The paper demonstrated the use of customdesigned charts and tables for estimating the daily collectable insolation by a solar collector that may be occasionally shaded by external horizon obstruc-

Table 3: Calculation of daily insolation for a partially shaded collector

\begin{tabular}{|c|c|c|c|c|c|c|c|c|c|c|}
\hline $\begin{array}{c}\text { Hour } \\
\text { centred } \\
\text { at: }\end{array}$ & $r_{d}$ & $r_{h}$ & $\begin{array}{c}I_{d} \\
\left(M J / m^{2}\right)\end{array}$ & $\begin{array}{c}I_{h} \\
\left(M J / m^{2}\right)\end{array}$ & $\begin{array}{c}\left(I_{h}-I_{d}\right) \\
\left(M J / m^{2}\right)\end{array}$ & $R_{b}$ & $F_{d}$ & $\begin{array}{c}I_{\text {coll }} \\
\text { Un-shaded } \\
\left(\mathrm{MJ} / \mathrm{m}^{2}\right)\end{array}$ & $s$ & $\begin{array}{c}I_{\text {coll }} \\
\text { Shaded } \\
\left(M J / m^{2}\right)\end{array}$ \\
\hline (solar time) & Tab.2 & Tab.2 & $r_{d} * H_{d}$ & $r_{h} * H_{h}$ & & Fig. 4 & Fig. 5 & Eq. 1 & Fig. 3 & Eq. 2 \\
\hline $6: 00$ & 0 & 0.00 & 0 & 0 & 0.00 & 2.00 & 0.98 & 0.00 & 1.00 & 0.00 \\
\hline 7:00 & 0.02 & 0.02 & 0.09 & 0.25 & 0.16 & 2.00 & 0.98 & 0.41 & 1.00 & 0.09 \\
\hline $8: 00$ & 0.059 & 0.05 & 0.27 & 0.85 & 0.58 & 1.47 & 0.98 & 1.12 & 0.50 & 0.69 \\
\hline 9:00 & 0.092 & 0.09 & 0.42 & 1.49 & 1.07 & 1.32 & 0.98 & 1.82 & 0.00 & 1.82 \\
\hline $10: 00$ & 0.118 & 0.12 & 0.53 & 2.06 & 1.53 & 1.26 & 0.98 & 2.45 & 0.00 & 2.45 \\
\hline $11: 00$ & 0.134 & 0.15 & 0.61 & 2.45 & 1.84 & 1.23 & 0.98 & 2.86 & 0.00 & 2.86 \\
\hline $12: 00$ & 0.139 & 0.16 & 0.63 & 2.59 & 1.96 & 1.22 & 0.98 & 3.01 & 0.50 & 1.81 \\
\hline $13: 00$ & 0.134 & 0.15 & 0.61 & 2.45 & 1.84 & 1.23 & 0.98 & 2.86 & 1.00 & 0.60 \\
\hline $14: 00$ & 0.118 & 0.12 & 0.53 & 2.06 & 1.53 & 1.26 & 0.98 & 2.45 & 1.00 & 0.52 \\
\hline $15: 00$ & 0.092 & 0.09 & 0.42 & 1.49 & 1.07 & 1.32 & 0.98 & 1.82 & 0.80 & 0.69 \\
\hline $16: 00$ & 0.059 & 0.05 & 0.27 & 0.85 & 0.58 & 1.47 & 0.98 & 1.12 & 1.00 & 0.26 \\
\hline $17: 00$ & 0.02 & 0.02 & 0.09 & 0.24 & 0.15 & 2.00 & 0.98 & 0.39 & 0.50 & 0.24 \\
\hline 18:00 & 0 & 0.00 & 0 & 0 & 0.00 & 2.00 & 0.98 & 0.00 & 1.00 & 0.00 \\
\hline Sum & & & 4.46 & 16.78 & & & & 20.30 & & 12.04 \\
\hline
\end{tabular}


tions. Although the construction of the said charts and tables requires expert knowledge in solar geometry and meteorology, once constructed, their usage in a tilted-plane sky model, for evaluating collectable insolation, does not require any such special expertise. The charts and tables are convenient for use by the average solar energy designer who need not have background training in the intricacies of solar geometry and meteorology. In the present case, the charts and tables have been designed to be applicable in Zimbabwe and for the standard flat plate configuration- equator facing and tilt equal to latitude, but they can also be designed for any other location and collector configuration.

The charts and tables are constructed using standard validated formulae, and to the extent that data can be accurately interpolated from them, they should produce dependable results. The only serious simplification is the use of a generalized radiation view factor for calculating diffuse radiation contribution. The resultant error has been analysed in the Appendix, and it was concluded that it would be negligible for most probable cases involving low-tilted collectors.

\section{References}

Collares-Pereira, M \& Rabl, A. 1979. The average distribution of solar energy -correlation between diffuse and hemispherical and between daily and hourly insolation values, Solar Energy 22(3): 155-164.

Crawford. T M.1995. Passive Solar Design Using Computer Generated Suncharts. Available online at: http://t016.arch.cf.uk/local/shading/Suncharts.htm, (accessed 12 October 2005).

Dennis, M K.2002. An automated solar shading calculator, In Proceedings 40th Annual Conference for the Australian New Zealand Solar Energy Society, Newcastle, NSW.

Duffie, J A. \& Beckman, W A.1991. In Solar Engineering of Thermal Processes, John Wiley \& Sons, New York: 32-40.

Erbs, D G, Klein, S A, Duffie J A. 1982. Estimation of the diffuse radiation fraction for hourly, daily and monthly averaged global radiation. Solar Energy 28 (3): 155-164.

Hottel, H C \& Sarofim, A F. 1967. In Radiative Transfer. New York: McGraw Hill: 31-39.

Hove, T \& Goettsche J. 1999. Mapping global, diffuse and beam solar radiation over Zimbabwe. Renewable Energy 18(6): 535-556.

Klein, S A. 1977. 'Calculation of Monthly Average Insolation on Tilted Surfaces', Solar Energy 19 (4): 325-329.

Klein, S A. 1996. A transient system simulation program, Solar Energy Laboratory, University of Wisconsin, Madison.

Liu, B Y H \& Jordan, R C. 1963. The long-term average performance of flat plate solar energy collectors. Solar Energy 7(2): 53-74.

Page, J K. 1961. The estimation of monthly mean values of daily total short-wave radiation on vertical and inclined surfaces from sunshine records for latitudes $40^{\circ} \mathrm{N}-40^{\circ} \mathrm{S}$. In Proceedings of the U.N. Conf. On New Sources of Energy, New York.

Rabl, A. 1985. In Active Solar Collectors and Their Applications, Oxford University Press, New York: 2835.

\section{Appendix: Analysis of error caused by using a generalised view factor}

In Section 5, in order to simplify the calculations, the diffuse radiation contribution was estimated by using a generalised view factor, $F_{d}$, equal to the view factor for an un-shaded collector. In fact, the view factor for the shaded collector is lower than that for the un-shaded case because part of the sky dome is masked from the collector view by the obstructing object. The error, in collectable radiation, introduced by using a generalized view factor instead of the correct factor is analysed in this Appendix.

A fairly extensive obstruction need to be analysed in order to get a confident judgment of the magnitude of error incurred. The collector site shown in Figure A1 was selected to facilitate the error analysis. The collector is located at the centre (point X) of a semi-circular wall of arbitrary radius and height such that the elevation angle of the wall skyline measured from the collector site is $\alpha_{0}$. The collector is facing North and has a tilt angle $\beta$.

Assuming that the distribution of diffuse radiation over the sky dome is isotropic, then the fraction of hemispherical diffuse radiation received at the collector site is proportional to the fraction of the sky dome 'seen' by the collector site. The total area of the sky dome can be calculated by integrating the standard unit sphere incremental surface area $\Delta \mathrm{A}$ (=sin $\alpha \mathrm{d} \alpha \mathrm{d} \gamma$ ) over hemisphere (see Figure A2). This integral reduces to $2 \pi$, the surface area of a hemisphere of unit radius.

For the case in Figure A1, the diffuse radiation originating from the part of the sky dome below the collector plane is not seen by the collector; and so is the part of the sky dome below the wall elevation angle ao and between azimuth limits 0 and $\pi$ (see Figure A3). The area of the sky dome seen by the collector, Ad, is therefore given by the integral:

$$
A d=\int_{0}^{\pi} \int_{x_{0}}^{\pi / 2} \sin \alpha d \alpha d \gamma+\int_{0}^{\pi} \int_{\pi / 2}^{\pi-\beta} \sin \alpha d \alpha d \gamma
$$

This definite integral reduces to $\pi\left(\cos \alpha_{o}+\cos \beta\right)$. 


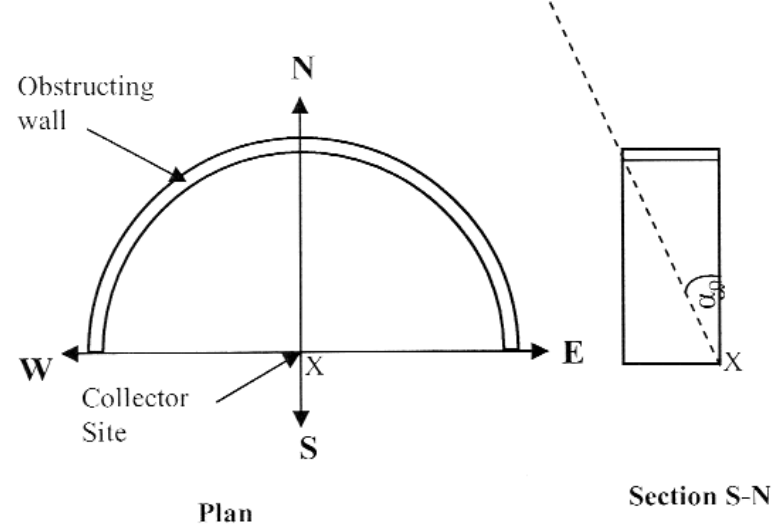

Figure A1: Plan and section of collector site

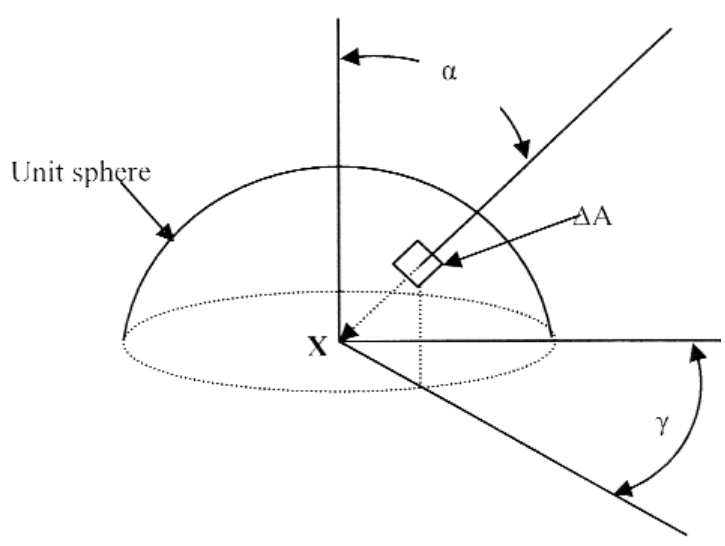

Figure A2: Sky dome represented by unit sphere

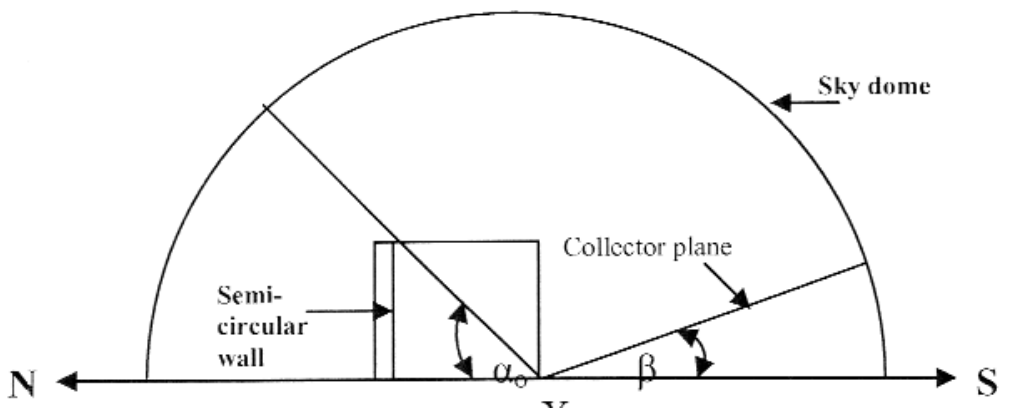

Figure A3: Schematic of collector site and sky dome

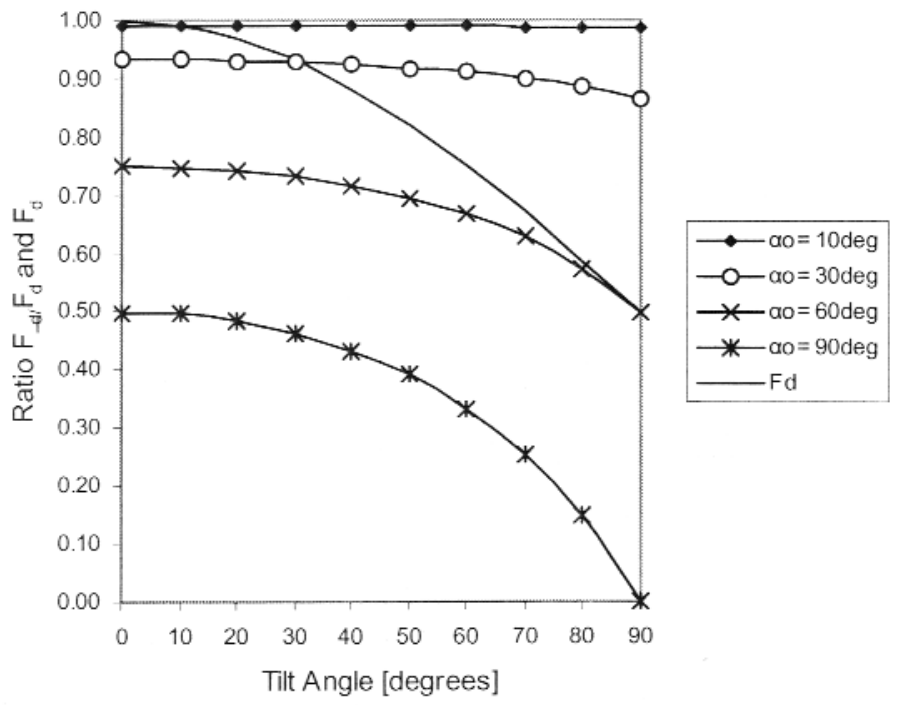

Figure A4: Variation of $\hat{F}_{d} / F_{d}$ with $\alpha_{o}$ and $\beta$; and of $\boldsymbol{F}_{d}$ with $\beta$

The fraction of the sky seen by the shaded collector - the is view factor for the shaded site, $\hat{F}_{d}-$ is obtained by dividing the definite integral of Equation (A1) by the surface area of the hemisphere, $2 \pi$, to give:

$$
\hat{F}_{d}=1 / 2\left[\cos \alpha_{o}+\cos \beta\right]
$$

The special case for a collector site that is not shaded can be obtained from Equation (A2) by putting $\alpha_{o}=0$ to give $F_{d}=1 / 2[1+\cos \alpha]$ as mentioned in Section 5. Also if both $\alpha_{o}$ and $\beta$ are equal to zero, a horizontal collector that is not shaded is represented, and $\hat{F}_{d}=1$ as expected. The ratio $\hat{F}_{d} / F_{d}$ is the required indicator of the level of error committed in assuming that the view factor is always equal to $F_{d}$. It is equal to $\left[\cos \alpha_{o}+\cos \beta\right] /[1+\cos \beta]$ and is plotted in Figure A4 for different values of $\alpha_{0}$ and $\beta$.

It can be seen from Figure A4 that the error committed in assuming that the view factor is $F_{d}$ 
Table A1: Percentage error in collectable radiation incurred by using Section 5 method for $\beta=20^{\circ}, s=1, R_{b}=1, I_{h}=1 \mathrm{MJ} / \mathrm{m}^{2}, I_{d} / l_{h}=0.25$

\begin{tabular}{lcccccccccc}
\hline${ }^{\circ}$ (degrees) & 0 & 10 & 20 & 30 & 40 & 50 & 60 & 70 & 80 & 90 \\
\hline \% error & 0.0 & 0.2 & 0.8 & 1.7 & 3.0 & 4.7 & 6.7 & 9.0 & 11.6 & 14.4 \\
\hline
\end{tabular}

increases as both ao and $\mathrm{b}$. For small tilt angles, the ratio $\hat{F}_{d} / \mathrm{Fd}$ varies from 1 to 0.5 as the angle of elevation of the obstruction, ao, varies from 0 to $90^{\circ}$. Taking the worst case of an improbable angle of elevation of $90^{\circ}$, and assuming $\mathrm{R}_{\mathrm{b}}=1, \mathrm{~s}=0$, and that the diffuse ratio is 0.25 , one can estimate the limit of the magnitude of error incurred by using the assumptions of Section 5. For a collector tilted at 20o and for an hourly global radiation of $1 \mathrm{MJ} / \mathrm{m}^{2}$, Section 5 method gives I $\mathrm{coll}_{\text {_lshaded }}=0.99 \mathrm{MJ} / \mathrm{m}^{2}$, compared to $0.87 \mathrm{MJ} / \mathrm{m}^{2}$ obtained when the correct value of $\hat{F}_{d}$ taken from Figure A1 is used. This represents an overstating of the collectable radiation of $14 \%$ by Section 5 method. However, improbably excessive shading has been assumed in this example. As shown in Table A1, for most practical situations the error should be much smaller and the assumption of Section 5 is justified. Where the designer desires greater accuracy, it is also possible to use the exact value of the view factor calculated through equation $\mathrm{A} 1$, with appropriate integration limits, to replace the generalized view factor employed in Section 5.

Received 7 September 2005; revised 12 January 2006 